\title{
Editorial
}

\section{Robustness Issues in Fault Diagnosis and Fault Tolerant Control}

\author{
Jakob Stoustrup ${ }^{1}$ and Kemin Zhou ${ }^{2}$ \\ ${ }^{1}$ Section for Automation and Control, Department of Electronic Systems, Aalborg University, Fredrik Bajers Vej 7C, \\ 9220 Aalborg, Denmark \\ ${ }^{2}$ Department of Electrical and Computer Engineering, Louisiana State University, Baton Rouge, LA 70803, USA \\ Correspondence should be addressed to Jakob Stoustrup, jakob@es.aau.dk \\ Received 24 January 2008; Accepted 24 January 2008
}

Copyright (c) 2008 J. Stoustrup and K. Zhou. This is an open access article distributed under the Creative Commons Attribution License, which permits unrestricted use, distribution, and reproduction in any medium, provided the original work is properly cited.

Fault diagnosis and fault tolerant control have become critically important in modern complex systems such as aircrafts and petrochemical plants. Since no system in the real world can work perfectly at all time under all conditions, it is crucial to be able to detect and identify the possible faults in the system as early as possible so that measures can be taken to prevent significant performance degradation or damages to the system. Fault diagnosis is not relevant only for safety critical systems, but also for a significant number of systems, where availability is a major issue.

In the past twenty some years, fault diagnosis of dynamic systems has received much attention and significant progress has been made in searching for model-based diagnosis techniques. Many techniques have been developed for fault detection and fault tolerant control. However, the issue of robustness of fault detection and fault tolerant control has not been sufficiently addressed. Since disturbances, noise, and model uncertainties are unavoidable for any practical system, it is essential in the design of any fault diagnosis/fault tolerant control system to take these effects into consideration, so that fault diagnosis/tolerant control can be done reliably and robustly. The objective of this special issue is to report some most recent developments and contributions in this direction.

The special issue is initiated by a paper by P. Zhang and $S$. Ding, which gives a review of standard fault detection formulations, focusing on robustness issues for model-based diagnosis systems.

N. Liu and K. Zhou then study a number of robust fault detection problems, such as $\mathscr{H} / \mathscr{H}, \mathscr{H}_{2} / \mathscr{H}$, and $\mathscr{H} / \mathscr{H}$ problems, and it is shown that these problems share the same optimal filters. The optimal filters are designed by solving an algebraic Riccati equation.
The robust fault detection and isolation problem is studied in the paper by E. Mazars et al., where an $\mathscr{H}$ criterion is used, giving rise to a quadratic matrix inequality problem. A jet engine example is provided.

D. Campos-Delgado et al. suggest an active fault-tolerant control, and a design strategy is provided, which takes model uncertainty into account. The methods are illustrated for a DC motor example.

Systems that can be described by linear parameter varying models are considered by S. Grenaille et al. where robustness constraints are included in the design of fault detection and isolation filters. An illustration of the methods is given in terms of an application to a nuclear power plant.

A method for design of a diagnosis and a fault tolerant control system using an integrated approach is presented in the paper by S. Yang and J. Chen. The design is illustrated for a double inverted pendulum system.

M. Benini et al. present both a linear and a nonlinear fault detection and isolation scheme. This paper focuses on robust fault diagnosis for an aircraft model, and has extensive simulations.

The fault tolerant scheme proposed by R. Dionisio and J. Lemos is capable of stabilizing systems with intermittent sensor faults. The approach is based on reconstructing the feedback signal, using a switching strategy where a model is used in the intermittent periods.

A design method for actuator fault diagnosis is proposed in the paper by $Q$. Zhang, where the focus is to obtain robustness with respect to nonlinear sensor distortion. A numerical example is given.

The problem of designing fault tolerant control systems for networked systems with actuator faults is treated in the 
paper by Li et al. The proposed design method is demonstrated by a numerical example.

The notion of a reliability index is introduced by $\mathrm{H}$. Li et al., for monitoring fault tolerant control systems. The reliability is evaluated based on semi-Markov models, and the approach is applied to an aircraft model.

The final paper of the special issue by $\mathrm{N}$. Wu et al. addresses fault tolerant control of a distributed database system. The fault tolerant design relies on data redundancy in the partitioned system architecture. Robustness is represented by the introduction of additional states modeling delays and decision errors. The design is based on solving Markovian decision problems.

Jakob Stoustrup

Kemin Zhou 

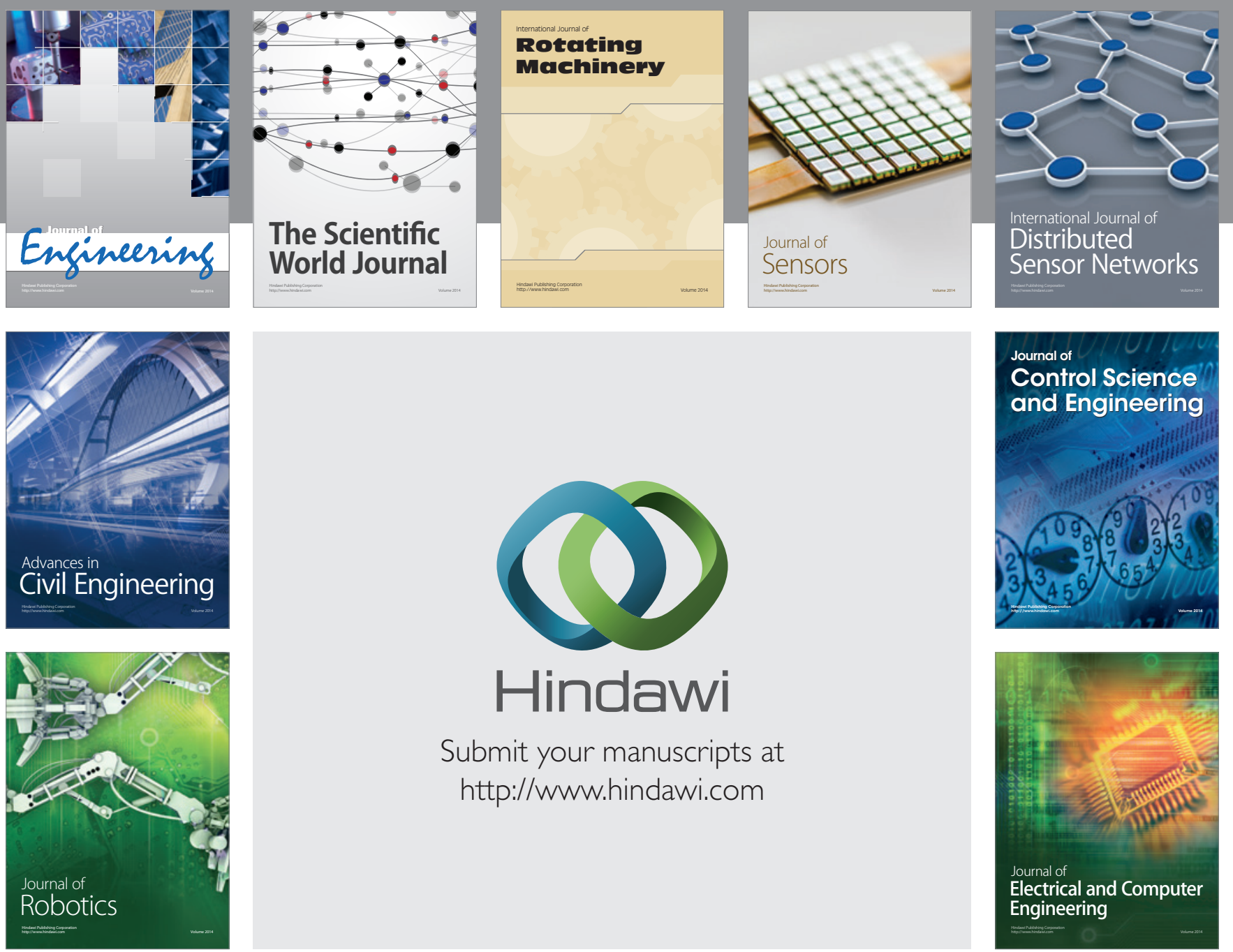

Submit your manuscripts at

http://www.hindawi.com
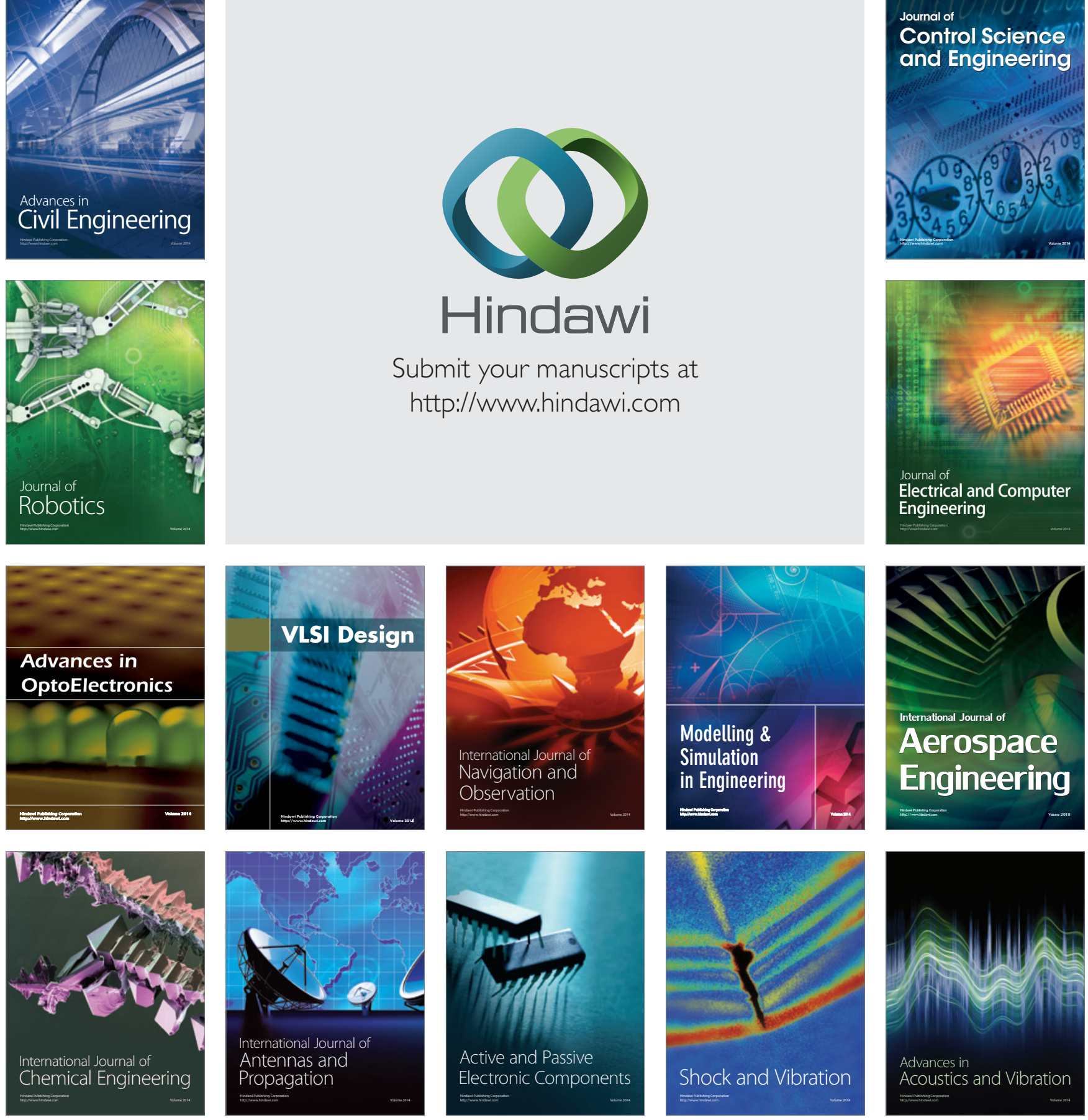